\title{
Cannabis infused foods and drinks: Food safety and the endocannabinoid system
}

\author{
Larry Holbrook $^{\mathrm{a}^{*}}$ and Michael Kani ${ }^{\mathrm{b}}$
}

${ }^{\mathrm{a} C B} 3$ Life Sciences Inc., 204-407 Downey Rd, Saskatoon SK, Canada, S7N 4L8

b210 Slimmon Rd, 40, Saskatoon SK, Canada, S7V 0L7

*Corresponding author: Larry Holbrook, CB3 Life Sciences Inc., 204-407 Downey Rd, Saskatoon SK, Canada, S7N 4L8.

E-mail: lah@cb3lifesciences.com

DOI: $10.31665 /$ JFB. 2018.4161

Received: October 25, 2018; Revised received \& accepted: November 29, 2018

Citation: Holbrook, L., and Kani, M. (2018). Cannabis infused foods and drinks: Food safety and the endocannabinoid system. J. Food Bioact. 4: 8-10.

\begin{abstract}
Until recently, consumer perception of cannabis was largely framed by its stigma surrounding drug use. Today, in an era of medical and recreational cannabis legalization, an increasing commercialization of cannabis-based products are being purported as functional foods. It is important for a wider audience of researchers, health care professionals, regulators and industry stakeholders to enhance their awareness of the human endocannabinoid system as a physiological regulator, the safety issues of product and process methods and adherence to qualitycontrolled training programs for assuring the public of safe practices.
\end{abstract}

Keywords: Endocannabinoid system; Cannabis edibles; CBD oils.

After decades of prohibition, today one can argue that cannabis has become mainstream from its use as medicine by the elderly to its use recreationally by a diverse population. With Canada becoming the second country besides Uruguay to legalize recreational use, this has led to a multitude of companies looking to cash in on cannabis value both as medicine and as food. The growing popularity to use parts of the plant as nutraceuticals raises numerous questions and concerns that requires examination by regulators, scientists, health professionals and all stakeholders. The interest here, is to bring more awareness to the research and food safety communities about the tide of activities accelerating in the realm of cannabis containing medicines, foods and recreational developments. Furthermore, advancing education and training for health care professionals about the human endocannabinoid system (ECS) is, in our view, a valuable enterprise.

With the World Health Organization (WHO) reviewing international drug scheduling of cannabis in November 2018 and having claimed in June for the non-scheduling of cannabidiol (CBD), the era of cannabis containing edibles and infused drinks sold with health claims or possibly as functional foods will likely grow even more exponentially. Intake of herbal compounds through ingestion, inhalation or topical application for the benefit of reducing pain and illness and/or attempting relief of discomfort for quality improvement of life is a cross cultural and evolutionary human behavior. For millennia, the cannabis plant has been a dominant example of humankind's earliest ventures into this medicinal and ritualistic use of plants. After a politically inspired pause in medicinal use of cannabis from Canada's 1923 "The Opium and Narcotic Drug Act" to the 1961 UN Convention on Controlled Substances, an intense revival since the 1990's is occurring with this plant for adjunctive utilization in medicine. Given a massive rise within just the past 5 years or so, especially in Canada, of oral medical preparations for ingestion, most recently a new generation of entrepreneurs is actively supplying cannabinoid actives in a vast array of ingestible products. This is further exemplified by cannabis infused products becoming discussion sessions in meetings like the Food Safety Consortium identifying safety plans, pre-requisite programs like GMPs, hazard analysis etc. There is also a plethora of cannabis trade shows with a vast array of edibles and drinks infused especially with non-psychoactive CBD. These would likely be considered functional foods containing bioactives. What might be the safety issues of production, processing and packaging and what is the level of education pertaining to awareness of the endocannabinoid system amongst this very diverse conglomerate of 


\section{innovators?}

In Canada, the term nutraceutical as opposed to functional foods does not have a legal definition. Nonetheless, Health Canada refers to them as any product that is isolated or purified from food generally sold in the form of medicine not associated with food and demonstrated to have physiological benefits or protection against chronic disease. Cannabis and its derivative cannabinoids, especially CBD is viewed by several stakeholders to fall under this reference. Studies using CBD had substantial higher amounts of CBD (e.g. 1,000 mg daily) to confer any therapeutic benefits (Pharmaceuticals, 2018). The CBD found in functional foods or nutraceutical products are far, far less but even assurance of validated quantitation is questionable. That's the difference between pharmaceutical and nutraceutical. Can these supplements therefore make any claims of effects?

The October 2018 initiation of legalized recreational Canadian adult use of cannabis is pushing the potential sales of many food and drink-based products with nefarious health claims and many questions remain as to informing about safety, production, and processing regulations. In essence, who provides and where is training available for cannabis product Quality Assurance, facility sanitation, global food safety initiative (GFSI) awareness, current Good Manufacturing Practices (cGMPs), Good Agricultural Practices (GAPs), HACCP, preventive controls qualified individuals (PCQI), chemical handling and safety? This is gaining more traction as the Safe Food for Canadians Regulations (SFCR) are set to come into effect on January 15, 2019.

Fortunately, even agencies like the American Society for Testing and Materials (ASTM) has a new Committee D37 for cannabis standardization of products and processes. This could help set harmonized procedures for regulatory agencies new to the medical cannabis arena. Other innovative approaches to gain knowledge about cannabis-based products include developing questionnaires and algorithms for data analysis to educate patients about healthrelated issues of such products (Klumpers and Thacker, 2018). Having this in mind, it is imperative to provide a wider audience awareness about food actives of cannabis containing products while acknowledging that the physiology reasoning behind this is a concurrent rising awareness and insight into the human endocannabinoid system.

To provide some background, botanically the cannabis plant is in the Family: Cannabaceae and most consistently considered dioecious as Cannabis sativa L. The female plant produces fatty acid containing resin from sessile and capitate trichomes rich in cannabinoids and terpenes, which provide the medicinal benefits. The resin contains delta-9-tetrahydrocannabinol (THC) known as the main psychoactive part of the plant, CBD which is not psychoactive, and there are estimates of more than 100 cannabinoids of which a few major dozen or so need more research to see if they have an active role working uniquely or synergistically with the already known compounds. A general review of hemp/cannabis biology is provided by Andre et al., (2016).

The continued rising wave of research into phytocannabinoids has been invigorated by the suggestion of patient experiences guiding the science. A research question in the early 1990's asked what the endogenous ligand might be for these phytocannabinoids such as $\Delta-9-$ THC and therefore a target for pharmacotherapy. The simple answer is that of an ancient, evolutionarily conserved and ubiquitous pleiotropic lipid signalling function in vertebrates known as the endocannabinoid system (ECS). Extensive reviews for the ECS are provided by Maccarrone et al., (2015) and Ligresti et al., (2016), plus more lipid chemistry associations in Tsuboi et al., (2018). The ECS is perceived to be a physiological regulatory system for attempting to modulate homeostasis. The G-protein coupled cannabinoid $(\mathrm{CB})$ receptors, $\mathrm{CB} 1$ and $\mathrm{CB} 2$ are acted upon by the endogenous eicosanoids arachidonoyl ethanolamide (AEA) better known as anandamide, and 2-arachidonoyl glycerol (2-AG). These are generated on demand in the CNS from cellular plasma membrane derived phospholipids and opened up the novel idea of retrograde transmission at synapses in the CNS. Enzymes involved include: diacylglycerol lipase for $2-\mathrm{AG}$ and $\mathrm{N}$-arachidonoyl phosphatidylethanolamide (NAPE) phospholipase-D for AEA. Hydrolysis involves presynaptic monoacylglyceride lipase (MAGL) for 2-AG and postsynaptic fatty acid amide hydrolase (FAAH) for AEA. These synthetic pathways and lipid metabolism along with $\mathrm{CB}$ receptor turnover, activation and degradation are commonly referred to as "endocannabinoid tone" to describe the closeness to or diversion from homeostasis. Therefore, CB receptors respond to three ligand sources: the endocannabinoids AEA and 2-AG produced by our body; phytocannabinoids such as THC and CBD derived from the cannabis plant; and synthetic cannabinoids like Nabilone (Cesamet ${ }^{\circledR}$ ) or other molecular entities such as WIN55212-2 developed for cannabinoid receptor binding studies. The $\mathrm{CB} 1$ and $\mathrm{CB} 2$ receptors can be found singular such as $\mathrm{CB} 1$ on neurons of the CNS or in combination distributed among tissues and organ systems including adrenal glands, heart, lungs, ovaries, spleen, muscles and given this distribution, the ECS is implicated in several physiological and pathophysiological processes to bring about that homeostasis. Processes include inflammation, appetite, memory, immune function, pain, regulation of stress and emotional state, and digestion. This becomes the fundamental basis for cannabis use as medicine.

In this developing arena of cannabis active pharmaceutical ingredients (APIs) in edible products, there is also confusion about hemp oil and CBD oil. Within phytocannabinoid nomenclature, the term hemp (industrial) in Canada refers to cannabis cultivars that contain less than $0.3 \%$ dry weight THC with no regulation on the CBD component. However, CBD content in hemp may range between $<1 \%$ and $>5 \%$ at seed set. Cannabinoids can be found in all parts of the plant having trichomes including the leaves, flower bracts, and small stems, but clean seeds are devoid of them. Consequently, a product labeled 'hemp oil' would be non-regulated seed oil fats extracted from the hemp seed whereas 'CBD oil' refers to compounded products having more concentrated $\mathrm{CBD}$ extracts supplied in an oil matrix of any number of lipid-based components such as olive oil. Hemp seeds have been known for generations to be important nutritional supplements due to their oil content being about $80 \%$ of polyunsaturated fatty acids, alphalinolenic acid, linoleic acid and notably a small amount of gamma-linolenic acid. There is also a substantial amount of vitamin E. Hemp seeds are often used as feeds for animals but the products they generate including oil, flour, protein powder are gaining popularity as an excellent food source for humans (Frassinetti et al., 2018).

Given the importance of the ECS in this physiological regulatory role, there is a strong basis in current Canadian production practices for licenced producers (LPs) to implement reasonably safe product and process controls. Although not required to be of a GMP standard there is Good Productions Practices (GPP) and many larger LPs do put GMP practices in place in anticipation of international markets. There are requirements for plant material testing of bacterial/ fungal contaminants such as salmonella, E coli pathogens, aspergillus, mycotoxin/aflatoxin analyses, heavy metals, solvent residues and detection of specific pesticides. Cannabinoids and terpenes are readily quantifiable although there continues to be efforts in harmonized, analytic methods for validation and compliance. As the industry grows and many subcontractors play roles in specific areas like chemical analysis only, not com- 
plete vertical integration within an LP, then procurers of raw materials will need to address and audit capabilities of these resources. This includes issues such as vetting supplier quality such as APIs, packaging certification as food safe, certificate of compliance or conformance, safety of excipients, Certificate of Analysis (CofAs), residual solvent, all documentation SOPs and open to audits by the purchasing agency.

Cannabis, unlike most other nutraceuticals or functional foods is different. Different in its use as both food and medicine and the role of the ECS. The overall concern of rapid expansion of a massive new market of cannabinoid containing foods and drinks is that producers/suppliers have an awareness and education in the pleiotropic physiological effects of the EC system, are trained in safe product processing/manufacturing practices, and do not make unsubstantiated claims for health benefits without clear evidence. Furthermore, there is a great deal of research potential open for lipid chemists, food scientists, healthcare professionals and others to delve into advancing knowledge of this lipid signalling EC system that has such a physiological impact.

\section{References}

Andre, C.M., Hausman, J-F., and Guerriero, G. (2016). Cannabis sativa: The
Plant of the Thousand and One Molecules. Front. Plant Sci. 7(Article19): 1-17.

Frassinetti, S., Moccia, E., Caltavuturo, L., Gabriele, M., Longo, V., Bellani, L., and Giorgetti, L. (2018). Nutraceutical potential of hemp (Cannabis sativa L.) seeds and sprouts. Food Chem. 262: 56-66.

Pharmaceuticals, G.W. (2018). A randomized, double-blind, placebo-controlled study to investigate the efficacy and safety of cannabidiol (GWP42003-P; CBD) as adjunctive treatment for seizures associated with Lennox-Gastaut syndrome in children and adults. https://clinicaltrials.gov/ct2/show/NCT02224560.

Klumpers, L.E., and Thacker, D.L. (2018). Development of a data driven tool to educate patients on efficiency and tolerability of cannabisbased products. 28th Annual Symposium on the Cannabinoids International Cannabinoid Research Society, Research Triangle Park, NC, USA, p. 51.

Ligresti, A., De Petrocellis, L., and Di Marzo, V. (2016). From phytocannabinoids to cannabinoid receptors and endocannabinoids: Pleiotropic physiological and pathological roles through complex pharmacology. Physiol. Rev. 96: 1593-1659.

Maccarrone, M., Bab, I., Bíró, T., Cabral, G.A., Dey, S.K., Di Marzo, V., Konje, J.C., Kunos, G., Mechoulam, R., Pacher, P., Sharkey, K.A., and Zimmer, A. (2015). Endocannabinoid signaling at the periphery: 50 years after THC. Trends Pharmacol. Sci. 36(5): 277-296.

Tsuboi, K., Uyama, T., Okamoto, Y., and Ueda, N. (2018). Endocannabinoids and related $\mathrm{N}$-acylethanolamines: biological activities and metabolism. Inflam. Regener. 38-28: 1-10. 